\title{
Criminologie
}

\section{Entre le retrait et la contestation. Réactions des mormons fondamentalistes à des allégations d'entorses aux lois}

\section{Marie-Andrée Pelland et Dianne Casoni}

Volume 41, numéro 2, automne-hiver 2008

Les organisations dites sectes, les lois et la société

URI : https://id.erudit.org/iderudit/019438ar

DOI : https://doi.org/10.7202/019438ar

Aller au sommaire du numéro

Éditeur(s)

Les Presses de l'Université de Montréal

ISSN

0316-0041 (imprimé)

1492-1367 (numérique)

Découvrir la revue

Citer cet article

Pelland, M.-A. \& Casoni, D. (2008). Entre le retrait et la contestation. Réactions des mormons fondamentalistes à des allégations d'entorses aux lois.

Criminologie, 41(2), 213-245. https://doi.org/10.7202/019438ar
Résumé de l'article

L'objectif de cet article est de comprendre les effets sur un groupe de mormons polygames fondamentalistes établis à Bountiful, en Colombie-Britannique, d'allégations d'entorses aux lois. Ces allégations vont du trafic de jeunes femmes entre le Canada et les États-Unis afin de les marier à des hommes polygames plus âgés qu'elles aux sévices physiques et sexuels sur des enfants, des adolescents et des femmes. Des entretiens avec des membres de ce groupe ainsi que l'analyse documentaire du journal de la communauté, des échanges sur le forum de discussion Web du groupe et des principaux journaux et périodiques canadiens et étatsuniens de 1990 à 2006 ont permis de documenter les allégations et de cerner les réactions du groupe. Celles-ci sont distinguées en deux périodes, un premier temps où le groupe a réagi par un retrait silencieux, puis un second où la contestation a dominé. Ces réactions sont décrites, analysées et illustrées à l'aide d'extraits d'entretiens et d'échanges Web. 


\title{
Entre le retrait et la contestation. Réactions des mormons fondamentalistes à des allégations d'entorses aux lois
}

\author{
Marie-Andrée Pelland \\ Professeure \\ Département de sociologie, Université de Moncton \\ marie-andree.pelland@umoncton.ca \\ Dianne Casoni \\ Professeure \\ École de criminologie, Université de Montréal \\ dianne.casoni@umontreal.ca
}

RÉSUMÉ - L'objectif de cet article est de comprendre les effets sur un groupe de mormons polygames fondamentalistes établis à Bountiful, en Colombie-Britannique, d'allégations d'entorses aux lois. Ces allégations vont du trafic de jeunes femmes entre le Canada et les États-Unis afin de les marier à des hommes polygames plus âgés qu'elles aux sévices physiques et sexuels sur des enfants, des adolescents et des femmes. Des entretiens avec des membres de ce groupe ainsi que l'analyse documentaire du journal de la communauté, des échanges sur le forum de discussion Web du groupe et des principaux journaux et périodiques canadiens et étatsuniens de 1990 à 2006 ont permis de documenter les allégations et de cerner les réactions du groupe. Cellesci sont distinguées en deux périodes, un premier temps où le groupe a réagi par un retrait silencieux, puis un second où la contestation a dominé. Ces réactions sont décrites, analysées et illustrées à l'aide d'extraits d'entretiens et d'échanges Web.

\section{Introduction}

Les mormons ont fait l'objet d'allégations d'entorses aux lois depuis la fondation du groupe en 1830 par Joseph Smith ${ }^{1}$ (Quinn, 1994 ; Bistline,

1. Les mormons ont fondé en 1830 la Church of Jesus Christ of Latter Day Saints, qui a abandonné la pratique de la polygamie en 1904 afin de se soumettre au Code criminel des 
2004). Par exemple, la presse canadienne a publié plus de quatre cents articles depuis 1990 dénonçant la pratique illégale de la polygamie, le trafic de jeunes filles entre les États-Unis et le Canada dans le but de marier ces adolescentes à des hommes plus âgés, le défaut de fournir une scolarisation suffisante aux enfants et de les protéger de la maltraitance et de l'agression sexuelle subies au sein de leur communauté. Ces allégations sont aussi relevées dans sept rapports commandés par des organismes gouvernementaux et de défense des droits de la personne. Ces rapports dénoncent tantôt la faible scolarisation des enfants et l'assujettissement des jeunes filles à une culture qui les contraint au mariage précoce et à la maternité répétée, tantôt la présence d'un mode de vie qui expose les enfants aux sévices physiques et sexuels, tantôt une situation sociale qui prive les femmes de leurs libertés fondamentales (Committee on Polygamous Issues, 1993; Brewin, 2004; British Colombia Teachers' Federation, 5 juillet 2004; Russell, 2004; Matas, 2005 ; Campbell et al., 2005; Cook, 2006). Enfin, depuis l'an 2000, le témoignage auprès des médias d'une femme ayant vécu à Bountiful de sa naissance jusqu'à ses 31 ans et que la publication subséquente en 2004 d'un livre présentant son expérience catapulte les mormons fondamentalistes de Bountiful à l'avant-scène en exposant les conditions de vie qui ont cours dans la communauté (Palmer et Perrin, 2004). Ainsi, depuis 1990, et avec une intensité renouvelée depuis 2000, les mormons de Bountiful font non seulement l'objet de multiples allégations de conduite illégale, mais aussi l'objet d'enquêtes de plusieurs agents de contrôle social, dont des agents de la GRC, des inspecteurs en bâtiments, des inspecteurs du ministère de l'Éducation et du ministère des Finances, des agents frontaliers et enfin des intervenants des services de protection de l'enfance.

À la lumière de la conclusion de Bromley (2002) selon laquelle ce sont précisément les interactions conflictuelles entre des représentants

États-Unis et de permettre l'inclusion de l'Utah au sein des États-Unis (Van Wagoner, 1989; Quinn, 1994). L'abandon de la pratique de la polygamie a donné lieu à une importante rupture chez les mormons. Plusieurs milliers de membres ont alors quitté l'organisation-mère pour fonder de nombreux groupes maintenant la pratique de la polygamie. Ils se sont désignés comme étant des fondamentalistes afin de souligner le fait qu'ils basaient leurs pratiques sur ce qu'ils considéraient comme les fondements de leur foi telle qu'elle se dégage d'une lecture textuelle de leurs livres sacrés, comme The Book of Mormon et Doctrine and Covenants. Les mormons qui se sont prêtés à cette étude font partie de ce dernier mouvement, connu sous le nom de Fundementalist Church of Jesus Christ of Latter Day Saints. Pour plus d'information sur l'histoire du mormonisme et du mormonisme fondamentaliste, voir Pelland et Casoni, 2009 (à paraître). 
d'institutions de contrôle social, d'une part, et, d'autre part, ceux de l'Ordre du Temple Solaire ainsi que ceux des Davidiens de Waco qui menèrent ces deux groupes à la violence autodestructrice, le présent article vise à cerner et à comprendre l'effet d'allégations d'entorses aux lois formulées à l'encontre des mormons fondamentalistes de Bountiful sur les membres de cette communauté. À la suite d'une brève recension d'écrits, la méthodologie utilisée sera décrite puis les allégations formulées contre la communauté de Bountiful et les réactions des membres à ces allégations seront présentées et analysées. La discussion des résultats permettra d'isoler deux réactions distinctes dans le temps, la première caractérisée par un retrait silencieux, suivie d'une réaction de confrontation principalement orchestrée par des femmes du groupe.

\section{Conflits entre groupes religieux minoritaires et société : ce qu'en disent les écrits}

La grande majorité des travaux actuels sur les conflits entre groupes dits sectaires et société proviennent de recherches rétrospectives et très peu d'études sont basées sur des données provenant de personnes vivant ou ayant vécu des conflits avec des représentants de la société. La recension présentée dans la présente section portera sur l'ensemble de ces écrits. En ce sens, la brièveté de cette recension est le reflet du peu de travaux qui portent sur la question des conflits entre groupes religieux minoritaires, dits sectes ou sectaires, et la société. Il est à noter que l'expression groupe dit sectaire a été choisie dans le cadre du présent article pour nommer ce que plusieurs auteurs désignent par le terme «secte» en raison de la connotation négative qui y est associée. Les expressions et les termes utilisés par les auteurs recensés seront cependant conservés dans la présentation de leurs travaux.

Pour Bromley (2002), Wilson (1991) et Wright (2002), la relation entre la société et les sectes, puisque ces dernières vivent en marge de la société, est inévitablement conflictuelle et cela d'autant plus que ces groupes rejettent activement nombre de valeurs et de normes par ailleurs acceptées par l'ensemble de la société. Wilson (1991) discute, à cet effet, de plusieurs types de situations qui favorisent l'émergence de conflits entre les sectes et la société. Parmi celles-ci, il indique les situations où le groupe religieux perçoit des acteurs sociaux extérieurs au groupe comme une menace pour l'intégrité du groupe, des personnes 
y vivant ou pour leurs valeurs et leurs normes. Plusieurs auteurs (Hall et Schuyler, 2000; Casoni, 2000; Hall et al., 2000; Bromley, 2002) observent que les représentants de la société et les groupes dits sectaires s'influencent entre eux dans la construction du conflit qui les oppose. Ce type de processus peut conduire à des épisodes tragiques de violence, notent-ils. Bromley (2002), Hall (1995) et Wright (2002) remarquent, à ce sujet, que plus les différences idéologiques entre les groupes dits sectaires et la société sont marquées, plus l'émergence de conflits entre les deux groupes est probable.

En cas de conflits intergroupes, le développement d'une vision stéréotypée de l'autre est fréquente. Cette construction est motivée, selon Hogg (2001), par le désir de sauvegarder une estime personnelle positive malgré les attaques dont les membres du groupe peuvent se sentir l'objet. Ellemers et al. (2002), Hogg (2001) ainsi que Smurda et al. (2006) sont d'avis que plus l'identité sociale d'un groupe est menacée, plus la vision stéréotypée de l'autre sera marquée. Enfin, plus le groupe religieux perçoit le conflit qui l'oppose à la société comme le menaçant, plus ses membres auront tendance à percevoir les autres sous un angle désavantageux (Hall et al., 2000; Robbins, 2002). Toutefois, il est à noter que, dans certaines circonstances, le sentiment d'être l'objet de persécution de la part de la société nourrit un sentiment de supériorité chez les membres du groupe désigné, comme le soulignent Mayer (2001) et Lifton (1999). La persécution dont ils se sentent l'objet peut donc devenir une source de valorisation pour le groupe dit sectaire, comme s'il s'agissait d'une confirmation de leur valeur et de l'importance de leur mission.

Selon plusieurs auteurs (Latkin, 1991; Bromley, 2001, 2002; Barker, 2002; Wright, 2002), les ex-membres de sectes, les associations dites antisectes et les groupes d'intérêts sont au nombre des acteurs qui peuvent influencer l'interaction conflictuelle opposant un groupe dit sectaire et les représentants de la société. Ces acteurs participent souvent à la stigmatisation du groupe, notamment en définissant leur mode de vie comme un problème social nécessitant l'intervention étatique. Nombre d'auteurs (Jenkins 1994; Randall-Noblitt et Penskin, 2000) signalent que les campagnes médiatiques de sensibilisation aux dangers que représenteraient les groupes dits sectaires ainsi que la médiatisation des violences perpétrées par quelques-uns de ces groupes contribuent au développement de représentations d'eux les faisant voir comme dangereux. Cette perception peut parfois même conduire les agents de 
contrôle social à intervenir prématurément ou sans raison valable (Bromley, 2002).

Devant les allégations formulées à leur encontre ou les enquêtes d'agents de contrôle social, plusieurs types de réaction ont été relevées (Larkin, 1991; Bromley, 2002; Wright, 2002). Certains groupes réagissent par le retrait - le cas du Peoples Temple fuyant les États-Unis pour s'établir à la Guyane en est un bon exemple -; d'autres cherchent à mieux s'intégrer à la société, comme les Témoins de Jehovah le font; certains autres groupes réagissent par la confrontation, comme les Davidiens l'ont fait à Waco, alors que d'autres encore ont recours à la contestation, comme le font les Scientologues, notamment en portant leurs détracteurs devant les tribunaux civils. La question de savoir comment un groupe précis, vivant au Canada et faisant l'objet de multiples allégations d'entorses aux lois, réagit à ces allégations est d'un intérêt certain non seulement en ce que cela peut contribuer aux connaissances sur les groupes religieux minoritaires en relation avec la société qui les entoure, mais aussi en ce que cela peut nous instruire sur les façons de mieux interagir en cas de conflits.

La présente étude fait partie d'une recherche plus large visant à comprendre comment les groupes religieux minoritaires ou dits sectaires et leurs membres réagissent aux allégations d'entorses aux lois. Cet article vise à cerner la réaction du groupe de mormons fondamentalistes dirigé par Winston Blackmore aux allégations qui leur sont faites dans les médias écrits. Deux questions forment la base des réflexions contenues dans cet article: comment les allégations à leur endroit affectent-elles les membres? Comment y réagissent-ils? Bien entendu ces questions en soulèvent d'autres, notamment en ce qui concerne la question de l'identité sociale et de ses vicissitudes. Il serait trop long toutefois d'aborder ces questions dans le cadre du présent article, qui sont analysées dans un autre document (Pelland et Casoni, soumis).

\section{Stratégie méthodologique}

Une approche qualitative par étude de cas a été choisie afin de cerner la réaction des membres de la communauté de Bountiful aux allégations d'entorses aux lois formulées contre eux. D'abord, des entrevues ont été conduites auprès de membres de cette communauté de mormons fondamentalistes rencontrés à Bountiful en Colombie-Britannique. Les 
entrevues étaient individuelles et ont eu lieu au cours d'un séjour d'un mois en 2005. Lors de ce séjour, le récit de l'expérience de douze mormons fondamentalistes, dix femmes et deux hommes, a été recueilli. La stratégie de prise de contact a consisté à approcher le leader, Winston Blackmore, au moment où des femmes de la communauté invitaient citoyens et journalistes à un événement d'information sur leur mode de vie nommé le Summit on Polygamy. L'objet de la recherche lui a été communiqué tout comme son déroulement général. Ce dernier a consenti à ce que les membres qui le désirent puissent y participer. Il s'agit donc d'un échantillon de convenance, c'est-à-dire que des entretiens ont été conduits avec toutes les personnes âgées de plus de 18 ans membres de la communauté de mormons fondamentalistes de Bountiful qui ont accepté d'y participer. À la suite de l'invitation du leader, neuf femmes et un homme ont contacté l'intervieweuse pour participer à la recherche. De plus, deux personnes, un homme et une femme, ont été directement sollicités lors du sommet. Il a été beaucoup plus facile de rencontrer des femmes que des hommes, puisque l'intervieweuse était femme, possiblement en raison de la culture très patriarcale de la communauté. En effet, malgré la formulation expresse d'une demande auprès de plusieurs participants pour rencontrer des hommes, uniquement deux membres masculins ont répondu à l'invitation.

Les données d'entrevues ont été confrontées à deux types de données documentaires, soit aux informations contenues dans les North Star Chronicles, le journal publié par le groupe, ainsi qu'aux échanges entre les membres du groupe sur un forum de discussion Web. Donc, plus de mille deux cents pages de discussion sur Internet qui ont eu lieu entre janvier 2005 et septembre 2006, l'ensemble des trente-sept parutions du journal North Star Chronicles, publié entre 2003 et octobre 2006, ainsi que les transcriptions d'entrevues ont été analysées. De plus, les articles publiés entre janvier 1990 et septembre 2006 dans de grands quotidiens, des journaux et des périodiques canadiens et étatsuniens traitant de la communauté de Bountiful ont été recensés². Les archives

2. Au nombre des journaux canadiens et étatsuniens recensés, des articles ont été recueillis dans 23 journaux canadiens et sept journaux étatsuniens. Journaux canadiens: Brandon Sun, Canadian Christianity; Canada National Secular Society; Calgary Sun; Calgary Herald; Christian Week; The Economist; Edmonton Journal; Edmonton Sun; Forbes; Globe and Mail; Kootenai Valley Press; MacLean's; La Presse; The Associated Press; The Province; The Medical Post; The National Post; The Tyee; The Toronto Star; The Toronto Sun; Saturday Night; Vancouver Sun; Winnipeg Sun. Journaux étatsuniens: Arizona Republic; Los Angeles Times; Phoenix New Times; Provo Daily Herald; Rocky Mountain News; Salt Lake Tribune; The Telegraph. 
du Vancouver Sun et du journal The Province ont également été consultées puisque ces journaux ont été spécifiquement cités par les participants. La lecture des journaux canadiens et étatsuniens permet de constater que 417 articles ont été publiés entre janvier 1990 et septembre 2006 traitant des mormons de Bountiful dans 30 journaux et périodiques différents. Cependant, entre 1990 et 1999, seulement 26 articles traitant des mormons de Bountiful paraissent, alors qu'entre 2000 et 2006, 391 articles sont publiés à leur sujet.

La nette augmentation d'articles de journaux publiés entre 2000 et 2006 au sujet des mormons fondamentalistes semble être reliée à plusieurs facteurs. D'abord, une fenêtre est ouverte sur la vie dans cette communauté par le témoignage de Debbie Palmer paru dans plusieurs journaux canadiens. Puis, possiblement en réaction au témoignage de Debbie Palmer, Winston Blackmore, alors qu'il était encore le leader de tous les mormons fondamentalistes de Bountiful, accorde nombre d'entrevues aux journaux à partir de 2000. Enfin, plusieurs événements marquants font la manchette, par exemple, la scission du groupe en 2002, la lutte subséquente pour le contrôle de l'école de la communauté, le Bountiful Elementary and Secondary School, ainsi que l'ouverture et le financement d'une seconde école, Mormon Hill Elementary and Secondary School.

De plus, les rapports produits par les groupes d'intérêts, les associations de protection des droits de la personne ainsi que par tous les autres organismes et associations ayant produit des rapports au sujet de la communauté de Bountiful ont été colligés et analysés. Enfin, les écrits des leaders, des membres et des anciens membres de la communauté de Bountiful ont été aussi colligés et analysés ${ }^{3}$.

Les articles parus entre les années 1990 et 2000 ont été recueillis à l'aide de la base de données de Biblio Branché, des archives numériques du Globe and Mail, ainsi que des articles disponibles au Centre de documentation d'Info-Secte et de la bibliothèque de la ville de Creston. Les articles parus entre 2000 et 2006 ont été recueillis à partir des archives de chacun de ces journaux disponibles sur Internet, de la bibliothèque de Creston, du Centre de documentation d'Info-Secte et des bases de données Biblio Branché, Newslink et Newspapers Online.

3. Au nombre des écrits recueillis auprès des membres de la communauté, notons des documents écrits et audiovisuels privés obtenus des participants, ainsi que les documents publiés sur le site Internet de la communauté: www.sharethelight.ca. Au nombre des documents publiés et des commentaires émis par des anciens mormons de Bountiful, notons le livre de Palmer et Perrin (2004) et les déclarations d'anciens membres parues dans des journaux canadiens (Armstrong, 30 septembre 2004; Bramham, articles du Vancouver Sun de 2004; Elsworth, 5 août 2004; Euringer, 18 novembre, 2004). 
Le discours des participants, leurs contributions au forum de discussion Internet et les articles publiés dans le journal de la communauté ont constitué suffisamment de sources d'information pour qu'une saturation des données soit atteinte. Afin de procéder à l'analyse des entretiens, ils ont été transcrits intégralement puis lus et relus afin de dégager les thèmes présents. La même stratégie a été utilisée pour les documents écrits, soit les contributions au forum de discussion Web et les parutions des North Star Chronicles. Afin de dégager le contexte d'émergence des réactions des membres de la communauté de Bountiful, ces données ont été confrontées aux articles de journaux traitant du groupe ainsi qu'aux autres écrits véhiculant des allégations contre eux. Une fois l'analyse verticale de l'ensemble des données complétée, une comparaison inter-documents a été réalisée pour permettre de dégager les thèmes récurrents et tenter de répondre aux questions de recherche.

\section{Portrait des allégations}

Les allégations les plus fréquemment recensées concernent la pratique de la polygamie. Bien que le caractère illégal de cette pratique soit souligné, ce qui est particulièrement dénoncé est le fait que la polygamie enfermerait les femmes dans un système patriarcal qui nie leurs droits et libertés et qui favorise le mariage précoce d'adolescentes aussi jeunes que 13 ans avec des hommes polygames plus âgés. Selon Bramham, du Vancouver Sun, dans un entretien avec Debbie Palmer, l'inaction gouvernementale serait basée sur le fait que la polygamie est considérée comme un crime sans victime. Cette ex-membre considère cependant que la polygamie est au cœur des difficultés vécues tant par les femmes et les enfants que par nombre de jeunes hommes qui vivent à Bountiful (Bramham, 21 juillet 2004). L'organisation sociale y serait à ce point patriarcale que les mormons fondamentalistes sont qualifiés de «talibans» de l'Amérique du Nord selon certains journalistes (Stick, 5 mars 2002; Cart, 18 août 2002; Bramham, 4 juin et 21 juillet 2004). Les femmes sont décrites comme un objet d'échange et le mariage arrangé y est décrit comme l'ultime moyen d'assujettissement des femmes. Selon Frazier et Florio, (16 juillet 2005) et Dougherty (13 mars 2003), les femmes mormones fondamentalistes accepteraient de n'avoir aucun pouvoir sur leur vie dans l'espoir d'ainsi accéder au salut éternel en suivant les commandements de leur religion. 
Outre les allégations portant sur la position sociale des femmes polygames, la question de la protection des enfants est une des inquiétudes les plus vives qui se dégagent de la lecture des journaux et des rapports consultés. Selon Daphnee Bramham, journaliste au Vancouver Sun, la pratique de la polygamie serait intrinsèquement liée à la perpétration de nombre de sévices et de torts aux jeunes de Bountiful: "C'est la raison des sévices, des détournements de filles mineures, de l'expulsion des jeunes hommes non préparés et non scolarisés pour s'adapter à la vie dans la société élargie.» (Bramham, 21 juillet 2004: A3)

Au nombre des allégations traitant des conditions de vie des enfants qui sont présentées dans les journaux consultés, plusieurs articles abordent la question de la sous-scolarisation des enfants. Elles reprennent pour la plupart les avis de la British Columbia Teacher's Federation (BCTF) tel qu'ils sont présentés dans son rapport du 5 juillet 2004 et dans les nombreux communiqués de presse qu'elle a émis. Il est ainsi allégué que l'école de la communauté ne prépare pas adéquatement les enfants ni sur le plan scolaire, ni sur le plan social. D'abord sur le plan scolaire, plusieurs voix s'élèvent pour dénoncer la qualité de l'enseignement donné aux enfants. Ces allégations sont principalement portées par la BCTF (5 juillet 2004), par Audrey Vance, cofondatrice du groupe Altering Destiny through Education, un organisme de sensibilisation à l'importance de l'éducation (D'Amour, 27 juillet et 2 août 2004 ; Jacobs, 12 juin 2004), ainsi que par deux anciennes membres, Debbie Palmer (French, 12 juin 2004) et Jane Blackmore (Armstrong, 30 septembre 2004; Bramham, 15 décembre 2004; Euringer, 26 mai 2006). Il est allégué qu'en raison de l'embauche exclusive d'enseignants qui sont des membres du groupe ayant rarement plus qu'une douzième année de scolarité, les enfants ne reçoivent pas un enseignement comparable à celui des autres enfants de la Colombie-Britannique. Ils déplorent aussi que le taux d'obtention de diplôme de fin d'études y soit très bas, ce qui laisserait les jeunes avec peu de ressources s'ils choisissent de quitter la communauté. De plus, la BCTF déplore que les enfants et les adolescents aient peu de possibilités de fréquenter des jeunes hors de leur milieu social, ce qui les préparerait mal à l'intégration à la société s'ils quittaient le groupe. À ce sujet, le phénomène dit des lost boys, soit l'expulsion des adolescents en raison de la menace qu'ils représentent pour l'accès des hommes d'âge mûr au mariage avec des jeunes filles, est décrit dans la presse comme préoccupant, compte tenu du peu d'éducation de ces jeunes et leur préparation inadéquate à l'intégration 
de la société en dehors de leur communauté (Bramham, 15 juin 2004).

Outre, la sous-scolarisation, les allégations de maltraitance des enfants et des adolescents sont parmi les allégations les plus fréquemment recensées. En effet, tant dans les rapports d'enquête (Committee on Polygamous Issues, 1993 ; Matas, 2005; Cook, 2006) que dans la presse (Oziewicz, 9 décembre 2000, Bramham, 21 juillet 2004), le milieu de vie à Bountiful est décrit comme mettant les enfants et les adolescentes à risque d'agression sexuelle. Selon certaines anciennes membres, les sévices sexuels se produisent quotidiennement (Oziewicz, 9 décembre 2000; Dougherty, 13 mars 2003; Mac Queen, 13 décembre 2004; Euringer, 18 novembre 2004, 26 mai 2006; Armstrong, 27 octobre 2005). Certaines de ces ex-membres rapportent même avoir été mariées à l'homme qui les aurait auparavant violées (Dawson, 22 septembre 2000; Frazier et Florio, 16 juillet 2005). Une autre exmembre raconte dans son autobiographie avoir été plusieurs fois victime d'agressions sexuelles lors de jeux organisés par des garçons plus âgés qui surveillaient les plus jeunes (Palmer et Perrin, 2004). Elle y décrit, en effet, une organisation sociale dans laquelle les enfants sont très souvent laissés sans surveillance ou sous la surveillance d'enfants parfois à peine plus âgés qu'eux, ce qui contribue certes à les mettre à risque non seulement d'agressions sexuelles, mais aussi de mauvais traitements physiques, soutient-elle. Cette ancienne membre témoigne d'ailleurs avoir souffert de graves et fréquents sévices physiques, surtout aux mains d'une des ses mères, c'est-à-dire de l'une des épouses de son père (Palmer et Perrin, 2004). Ainsi, l'absence de surveillance parentale et une organisation sociale patriarcale sont vues comme la source des sévices physiques dont seraient victimes nombre d'enfants et d'adolescents de Bountiful (Remington, 27 septembre 2000; Dougherty, 13 mars, 10 avril et 21 août 2003; Bramham, 21 juillet 2004).

Parmi les allégations de maltraitance figure aussi l'exploitation sexuelle des adolescentes rendue aisée par leur mariage arrangé, le plus souvent avec des hommes beaucoup plus âgés qu'elles. Des anciens membres révèlent dans des entretiens avec des journalistes que les jeunes filles se marient généralement entre l'âge de 13 et 17 ans (Dawson, 22 septembre 2000; Remington, 27 septembre 2000; Bell, 12 juillet 2002; Bramham, 21 juillet 2004; Kent, 2006). Comme le fait d'avoir une famille nombreuse est vu comme nécessaire afin d'accéder au paradis, ces jeunes épouses sont souvent mères dès l'adoles- 
cence. À ce propos, Daphnee Bramham, du Vancouver Sun, précise que la communauté de Bountiful a plus que doublé sa population depuis 1990 (21 juillet et 7 août 2004), la fertilité des adolescentes de la communauté y contribuant probablement beaucoup (French, 12 juin 2004; Elsworth, 5 août 2004; Bramham, 7 août 2004). Certains journalistes soutiennent que ces mères adolescentes sont considérées comme les génitrices (breeding stock) du groupe (Dougherty, 21 août, 2003 ; Bramham, 29 mai 2004; Jacobs, 12 juin 2004; D'Amour, 2 août 2004).

Selon une autre allégation fréquente il existerait un trafic de jeunes filles entre les États-Unis et le Canada dans le but de marier ces adolescentes à des hommes polygames de Bountiful. Cette allégation provient principalement du récit d'anciens membres canadiens et étatsuniens et est rapportée tant dans la presse canadienne qu'étatsunienne (Dawson, 22 sept 2000; Woods, 4 août 2001 ; D'Amour, 2 août 2004; Bramham, 29 mai 2004, 13 octobre 2005). En effet, plusieurs journalistes soutiennent que, dans le but d'éviter les mariages consanguins, plusieurs adolescentes auraient été introduites illégalement au Canada afin d'y être mariées (Woods, 4 août 2001; Matas, 19 novembre 2002; Dougherty, 13 mars 2003; Weland, 24 novembre 2004). Pour Woods (4 août 2001), ce trafic aurait aussi pour fonction de gérer le cas d'adolescentes dites rebelles en les exilant loin de chez elles. Cette accusation a été émise pour la première fois en 2000 par le journaliste Fabian Dawson dans The Province, à la suite des déclarations de Craig Chatwig, un ancien membre de la Fundamentalist Church of Jesus Christ of Latter-day Saints (Dawson, 22 septembre 2000). Ce dernier alléguait que sa sœur, Esther Ruth, avait clandestinement passé la frontière canado-américaine peu après son $13^{\mathrm{e}}$ anniversaire pour y être mariée à un homme polygame de 28 ans de Bountiful qu'elle ne connaissait pas.

Depuis la déclaration de Chatwig (Dawson, 22 septembre 2000), un groupe canadien et étatsunien d'anciens membres a dénombré plus de cinquante jeunes filles qui auraient ainsi été introduites clandestinement dans l'un des deux pays. Ils rapportent aussi l'existence d'une route secrète assurant leur passage entre les frontières (Woods, 4 août, 2001 ; Bell, 12 juillet 2002). Ce trafic soulève également la question du statut précaire de ces jeunes filles clandestinement introduites au pays. En effet, comme elles se trouvent en situation d'illégalité, elles deviennent conséquemment très dépendantes de la communauté d'accueil, mais aussi très vulnérables en cas de mauvais traitements. 


\section{Actions entreprises}

En plus des allégations formulées par la voie des journaux et des témoignages d'anciens membres livrés à des journalistes, certains individus et groupes ont entrepris des démarches auprès de différents paliers de gouvernements ou ont fait des interventions publiques au sujet des communautés de mormons fondamentalistes de Bountiful. Voici un résumé de ces principales interventions. Debbie Palmer, ancienne membre de la communauté de Bountiful, fonde un groupe de pression dénonçant la polygamie nommé Committee Concerned with Polygamous Issues (Oziewicz, 9 décembre 2000). Elle adresse également une lettre à la ministre de la Justice du Parlement canadien, Ann McLellan, afin de solliciter son intervention. Elle demande aussi la modification de l'article 293 du Code criminel sur la polygamie afin que le gouvernement de la Colombie-Britannique puisse entamer des poursuites contre les dirigeants de la communauté de Bountiful. Le 11 juillet 2002, Debbie Palmer organise un forum réunissant des ex-membres de communautés polygames et des groupes de lutte contre la polygamie à Vancouver. Elle y déclare que la tolérance des autorités canadiennes favorise l'immigration de familles polygames au Canada (Bell, 12 juillet 2002; Cart, 18 août 2002).

En 2003, d'anciennes membres de la communauté de Bountiful s'unissent afin de poursuivre le gouvernement canadien pour leur inaction relativement à la communauté polygame de Bountiful malgré les preuves de la pratique ouverte de la polygamie cumulées par la Gendarmerie Royale du Canada. Elles poursuivent également le United Effort Plan, le fonds monétaire des mormons de la FLDS, pour enrichissement injuste (Nichols, 19 octobre, 2003; French, 12 juin 2004; Jacobs, 12 juin 2004, 21 mai 2006).

En 2004, neuf femmes, dont Debbie Palmer et Jancis Andrews, de la Canadian Federation of University Women, déposent une plainte au Tribunal des droits de la personne de la Colombie-Britannique. Elles accusent le procureur général de la Colombie-Britannique, le ministre de l'Éducation de cette province et les leaders de Bountiful de contrevenir aux droits des femmes de la communauté (Bramham, 29 mai 2004; Betty, 4 septembre 2004). À la suite de cette plainte, la Conférence des Églises Unies de la Colombie-Britannique - The B.-C. Conference of the United Church - encourage ses membres à écrire au procureur général de la province pour protester contre son inaction au 
regard des sévices apparemment commis dans la communauté de Bountiful (Chattaway, 7 juillet 2004). Également en réaction à cette plainte, la British Columbia Civil Liberties Association envoie à l'été 2004 une lettre au premier ministre de la Colombie-Britannique, Gordon Campbell, afin qu'il institue une enquête pour examiner l'ensemble des allégations formulées contre la communauté de Bountiful.

À la suite de la publication de son autobiographie, en décembre 2004, Debbie Palmer reçoit de nombreuses invitations pour discuter de son livre, notamment provenant de groupes de défense des droits des femmes. L'organisme West Coast LEAF organise ainsi une rencontre pour discuter du lien entre la pratique religieuse et l'atteinte aux droits et libertés. En février 2005, Beyond Borders, un organisme de défense des droits des enfants, organise une table ronde portant sur la prévention de sévices sexuels dans les communautés polygames. Cette rencontre donne lieu à la publication d'un rapport qui demande au gouvernement canadien de modifier l'âge du consentement aux relations sexuelles de 14 à 16 ans afin d'empêcher les jeunes adolescentes de se marier précocement dans la communauté de Bountiful (Matas, 2005). Il est espéré que, si les relations sexuelles avec une épouse de moins de 16 ans constituent une offense criminelle, cela incitera le clergé de Bountiful à surseoir au mariage des jeunes filles avant cet âge. En réponse à cette demande, le gouvernement fédéral présente un projet de loi à cet effet en 2006.

L'ensemble de ces interventions a entraîné une réaction sociale envers la communauté de Bountiful. Comme le précise Beaman (2004), la communauté est dorénavant perçue comme un environnement à risque pour les femmes et les enfants, d'où la nécessité d'ouvrir une enquête afin de vérifier le bien-fondé des nombreuses allégations et, le cas échéant, de prendre des mesures pour mieux les protéger. Pour certains, les mormons de Bountiful sont dorénavant perçus comme menaçant les valeurs canadiennes fondamentales, plus particulièrement la monogamie (Wood, 4 août 2001; Beaman, 2004; Elsworth, 5 août 2004).

\section{La réaction des mormons fondamentalistes de Bountiful}

Les participants réfutent les allégations de façon presque complète. Ils admettent qu'il a pu y avoir certains sévices sexuels commis dans le passé, mais que les personnes qui en étaient coupables ont été condamnées en 1990, ce qui clôt pour eux cette question. Pour ce qui en est 
des sévices physiques, les participants affirment avoir appris, il y a quelques années, que les punitions corporelles sont contre-indiquées pour le sain développement des enfants et ne plus y recourir depuis. Quant au mariage des jeunes filles, ils avouent que des adolescentes aussi jeunes que 15 ans aient pu, dans le passé, être mariées à des hommes plus âgés, mais que désormais aucune adolescente de moins de 18 ans ne pourra se marier avec un homme qui a déjà d'autres épouses quoique les jeunes filles pourront continuer d'être mariées à des jeunes hommes célibataires. Par ailleurs, ils réfutent complètement les autres allégations, disant qu'il s'agit de calomnies et de mensonges. Ils sont particulièrement durs envers Debbie Palmer, cette ancienne membre de Bountiful qui a publié son autobiographie en 2004, arguant qu'elle ne peut connaître ce qui se passe dans le groupe depuis son départ, il y a 18 ans, et en témoigner. Ils contestent, en outre, le statut d'«experte» du mormonisme que les médias lui attribuent. Ils déplorent que les médias accordent autant de crédit aux anciens membres et si peu à ceux qui y vivent et qui seraient donc en mesure de témoigner de leur réalité de façon plus juste. Plusieurs participants ont exprimé leur impression qu'il ne sert à rien, ou presque, de parler aux journalistes, ceux-ci étant plus enclins à publier des articles «sensationnalistes» que leurs réfutations des allégations à leur endroit.

La question qui se pose est de cerner si les allégations à leur endroit favorisent l'émergence de relations conflictuelles avec la société. Plus spécifiquement, comme le constatent Bromley (2002), Hall (1995) et Wright (2002), est-ce que les allégations qui pèsent sur cette communauté relativement fermée donnent lieu à des relations conflictuelles avec les agents de contrôle social? Cette question se pose d'ailleurs aussi par rapport aux médias.

Relations avec les institutions de contrôle social: conflit ou harmonie?

Selon les participants rencontrés, la publication d'allégations dans la presse canadienne a eu pour effet d'augmenter le contrôle étatique sur la communauté. Diverses institutions gouvernementales semblent ainsi utiliser les moyens à leur disposition pour évaluer le bien-être des enfants, le trafic de jeunes filles, ou encore la scolarisation des enfants. Lécole de la communauté est ipso facto évaluée annuellement et les contrôles fiscaux sont plus fréquents depuis 1990. Les agents de la GRC 
et des services de protection des enfants visitent les familles après la formulation de toute nouvelle allégation de mauvais traitement ciblant une famille ou un enfant donnés. Les allégations ont donc un effet direct sur la vie quotidienne des membres. Malgré l'augmentation des visites de contrôle, plusieurs participants de cette recherche suggèrent que non seulement les agents de contrôle social font leur travail adéquatement, ce qu'ils acceptent, mais aussi qu'ils sont, eux aussi, l'objet d'allégations sans fondement:

Nous avons eu une réaction immédiate de la GRC. Les services sociaux, ils ont l'habitude de visiter le groupe et d'enquêter sur les allégations. Ils sont venus et ils ont enquêté sur les allégations [sévices sexuels] et ils ont trouvé, à ma connaissance, deux cas. Les services sociaux seront les premiers à vous dire qu'ils sont extrêmement prudents avec cette information, ils sont très discrets. Ils ne divulgueront l'information à personne. Il peut y avoir plus de cas, je ne déclare pas ici qu'il n'y en avait pas d'autres, mais ils ont trouvé deux cas, ces deux cas ont fait l'objet de procédure judiciaire, ils ont été accusés de la même manière que tout le monde. Ça c'est l'une des frustrations que j'ai. La GRC et les services sociaux, ils connaissent notre existence, je suis certain que c'est frustrant pour eux parce qu'ils doivent faire face aux mêmes allégations. (Mark ${ }^{4}$, avril 2005)

Cet extrait illustre comment Mark cherche à présenter les agents de la GRC, ainsi que les intervenants des services sociaux, comme des alliés naturels, en ce que ceux-ci souffriraient, comme les mormons de Bountiful, du climat d'allégations à leur encontre. Par ailleurs, il est intéressant de noter que l'attitude sympathique décrite par Mark à l'endroit des agents de contrôle social ne témoigne pas du clivage que Hogg (2001) observe lorsqu'un groupe est en conflit avec la société qui l'entoure. Cela peut s'expliquer par le fait que, dans ce cas, en l'absence d'actions plus directes entreprises par les institutions de contrôle social, c'est avec les médias et les groupes d'intérêt que les mormons de Bountiful sont en conflit. Toutefois, il est à noter que non seulement ils ne se laissent pas entraîner dans une généralisation de leur conflit avec les médias et les groupes d'intérêt, mais ils cherchent de façon claire à faire alliance avec les agents de contrôle social, ce qui ne correspond nullement aux attitudes décrites par les auteurs ayant spécifiquement étudié de tels conflits avec des groupes de même nature (Latkin, 1991;

4. Dans le but de préserver l'anonymat des participants de la recherche, les prénoms utilisés pour les identifier sont fictifs. 
Bromley, 2001, 2002; Barker, 2002; Wright, 2002). Ce prochain extrait rend encore plus clairement le rapprochement expérientiel suggéré par les participants avec les agents de contrôle social:

L’abus [par les médias] est vraiment pernicieux, notre école a été évaluée plus souvent que n'importe quelle autre école. Nous avons été évalués par les dirigeants, et parce qu'ils ne trouvent rien, ils les attaquent. Le Procureur général parce qu'il n'est pas intervenu, ils l'attaquent. Notre maire, ils l'attaquent. (Eileen, avril 2005)

Sans parler d'alliance avec les instances gouvernementales, Eileen suggère néanmoins que les agents de contrôle social subissent les mêmes pressions que le groupe. L'image qui se dégage de ces propos en est aussi une image de victimisation indirecte, comme si le sous-texte suggérait que ces représentants de la société étaient victimes de harcèlement puisqu'ils constataient, à l'instar de ce que les mormons l'affirment, qu'il n'y avait pas matière à allégation. D'ailleurs, bien que les participants se voient comme des victimes de harcèlement, ils n'en attribuent pas la responsabilité aux agents de contrôle social, mais plutôt aux médias et à certains anciens membres:

Ils [les journalistes] arrivent avec leurs propres objectifs, ils ne veulent pas la vérité, ils veulent du sensationnalisme. Le sensationnalisme, c'est ce qui vend, donc c'est tout ce qu'ils veulent. Si nous disons quelque chose, nous ne le retrouvons pas dans le journal et je suis indignée de cela. (Kathleen, avril 2005)

Cet extrait témoigne de ce qui a été décrit ailleurs comme un effet de stigmatisation consécutive aux interventions dans les médias d'exmembres ou de groupes d'intérêt (Latkin, 1991; Bromley, 2001, 2002; Barker, 2002; Wright, 2002). En ce sens, les agents de contrôle social sont vus, contrairement aux journalistes, comme remplissant le devoir de leur fonction:

Ils [les agents de contrôle social] réagissent à celles-ci [allégations], ils enquêtent si quelqu'un leur donne un nom. Ils le font vraiment bien et ils ont toujours eu une attitude positive envers nous. (Margaret, avril 2005)

Cette représentation des agents de contrôle social comme victimes de harcèlement de la part des journalistes et des anciens membres a souvent été relevée, comme si cette façon de voir donnait un sens à l'expérience des membres, à tout le moins, en leur permettant de l'expliquer, sans toutefois être obligés de se remettre en cause pour autant. Il est inté- 
ressant de noter dans le prochain extrait comment Margaret désigne les agents de la Gendarmerie royale du Canada comme «nos agents» et «notre sergent en chef»:

Nos agents de la GRC sont sous surveillance constante. Tu sais: «Vous laissez ces événements se produire et vous ne faites rien!» En fait, notre dernier sergent-chef, celui qui a quitté la ville au printemps, il a été chef durant 14 ans, il aimait vraiment Creston, mais tout ce qu'il obtenait c'était du harcèlement, parce qu'il ne faisait pas son travail! Dans les faits, il a enquêté sur chacun des cas où il y avait un nom. Ils ont toujours fait leur travail. (Margaret, avril 2005)

Non seulement les rapports avec les agents de contrôle social sont-ils décrits comme courtois, mais dans les mots de Margaret, comme presque familiers. Ainsi, aucun entretien ne permet d'isoler quelque velléité de contestation ou de confrontation avec ces derniers. L'hostilité est plutôt dirigée vers les médias et vers les anciens membres. Cependant, il y a lieu de se demander si les agents de contrôle social susciteraient le même type de réactions si leurs enquêtes menaient à des accusations ou à des interventions plus répressives. Pour le moment, toutefois, ils sont plutôt vus avec sympathie, comme le sont aussi les politiciens:

Je pense quactuellement c'est une période horriblement contraignante pour nos politiciens, une période contraignante pour notre GRC, une période contraignante pour nos gouvernements, disant: «Vous savez, ce groupe polygame existe, il y a des hommes qui...» Ils amplifient hors de toute proportion. Aucun homme n'a 30 épouses, mais ils essaient de le laisser entendre afin d'engendrer la haine, spécialement les personnes qui ont quitté. Ils cultivent vraiment la haine à notre égard. (Margaret, avril 2005)

Non seulement les allégations sont vues comme malicieuses: «ils amplifient hors de toute proportion», mais elles sont aussi assimilées à une campagne de haine: «ils essaient de le laisser entendre afin d'engendrer la haine [...] Ils cultivent vraiment la haine à notre égard» (Margaret, avril 2005). La réaction de colère se dirige davantage vers «les personnes qui ont quitté» (Margaret, avril 2005) et épargne les politiciens en dépit du fait que le Procureur général de la ColombieBritannique, Geoff Plant, a commandé une enquête sur la communauté en faisant référence aux allégations de tort contre des membres de Bountiful qui sont soutenues par des anciens membres et diffusées par les médias. Dans les prochaines sections, les stratégies utilisées par les participants pour faire face au climat d'allégations qui marquent leur vie seront présentées. 


\section{Réactions aux allégations: de la tolérance passive à la proaction}

L'analyse du discours des membres de la communauté permet de constater que le groupe a adopté deux réponses différentes pour faire face aux allégations d'entorses aux lois. De 1990 à 2004, ils adoptent une stratégie de tolérance passive que nous avons désignée comme un retrait silencieux puis, avec l'intensification des allégations contre eux, ils adoptent, de 2004 à 2006, une stratégie proactive dite de contestation. Pour les participants, la distinction est évidente entre la réaction de retrait et celle de contestation:

Au cours de la dernière année, nous avons plus que jamais exposé nos vies à un examen minutieux, au regard de l'autre parce que nous sommes fatigués des allégations, tu sais. Avant, on croyait que le fait de rester entre nous, d'être silencieux, était la solution juste, mais depuis, silencieux nous ne sommes plus! (Judith, avril 2005)

\section{Le retrait silencieux: 1990-2004}

Entre 1990 et 2004, les participants disent avoir évité les interactions avec les gens extérieurs au groupe. Ils ne répondent pas aux allégations à leur encontre et croient qu'il s'agit là de la meilleure stratégie:

Ils disent: «C'est vraiment frustrant parce que, tu sais, vous êtes la seule que j'ai rencontrée qui peut gagner un argument en gardant le silence». Et si quelqu'un veut argumenter avec nous, ils n'ont nulle part où aller avec le silence! (Carole, avril 2005)

La persécution, je sens qu'elle n'était pas trop intense, mais c'est l'une des raisons pour lesquelles nous sommes restés silencieux et que nous sommes restés dans notre coin. (Nicole, avril 2005)

Une telle forme de réaction, soit un retrait devant des allégations perçues comme hostiles, a été notée par Bromley (2002). Les allégations sont en effet construites comme de nature persécutoire, même si Nicole parle d'une persécution qui n'était au départ pas trop intense. Le choix de ne pas répondre semble aussi constituer une réaction partagée qui consolide le groupe et renforce une impression d'être à la fois marginale et marginalisée, ce qui donne un sentiment de sécurité associé à la pérennité du groupe: «Le silence est le moyen par lequel nous avons préservé notre mode de vie». (Eillen, avril 2005) Wilson (1991) discute 
de situations où le groupe se sent menacé dans son essence même, ce qui apparaît bien être le cas ici. Carole semble tirer un sentiment de fierté de cette stratégie, comme si les membres étaient plus forts puisqu'ils sortaient gagnants de toute discussion en recourant au silence. Comme Mayer (2001) et Lifton (1999) l'ont déjà constaté, les membres se sentent entre 1990 et 2004 grandis par la persécution dont ils se reconnaissent être l'objet. La position de retrait se dégage clairement des extraits tirés des entretiens avec Eileen et Nicole. Mais aussi, il ressort que les allégations sont vues comme des manifestations de persécution décrites comme faisant partie d'une expérience normale et attendue:

Aussi, vous devez savoir que les allégations ont toujours été présentes. Même en Utah, dans le sud de l'Utah, j'ai été élevée là-bas et il y a toujours eu des allégations. (Mary, avril 2005)

[...] le mormonisme a débuté dans un nuage de persécution, c'est de cette façon depuis toujours. J'ai enseigné à mes enfants à s'attendre à l'être, parce que si vous croyez dans cette façon particulière, vous devez vous attendre à être persécuté. C'est une partie de notre expérience, ça a fait de nous qui nous sommes. (John, avril 2005)

L'expectative de la persécution est transmise par John à ses enfants comme faisant partie de l'expérience spirituelle même de leur religion. Ce qui est implicite dans son discours est que la persécution les rend plus forts ou même meilleurs qu'autrui: «ça a fait de nous qui nous sommes». Il n'est donc pas surprenant que la réaction première aux allégations à leur endroit ait été un retrait silencieux. Les participants disent à cet effet s'inspirer de l'expérience de leurs parents, qui tolérèrent la persécution avec stoïcisme. L'arrestation massive de mormons fondamentalistes en 1953 à Short Creek $^{5}$ est souvent citée comme une expérience fondatrice de leur religion à laquelle ils se doivent d'être à la hauteur:

Je pense que malgré le fait que je n'ai pas vécu durant cette période de persécution, je l'ai dans mon cœur et je sais ce que mes parents ont souffert pour me permettre d'être où je suis. (Nicole, avril 2005)

5. Lors du raid de Short Creek, maintenant connu comme la ville de Colorado City, le 26 juillet 1953, le shérif et ses hommes arrêtèrent 36 hommes adultes et quelques femmes sans enfant. De plus, 56 femmes et 153 enfants ont été déportés aux quatre coins de l'État de l'Arizona. Deux ans après le raid, tous les membres de la communauté étaient de nouveau réunis à Short Creek. 
La fierté d'être mormone fondamentaliste se dégage de ce court extrait, comme si les épreuves subies par ses parents lui imposaient un devoir de mémoire semblable à ce dont les enfants de survivants de l'holocauste expriment. Le leader actuel du groupe, Winston Blackmore, s'inspirant possiblement de la Bible, rappelle, à cet effet, un sermon de Leroy Johnson, un leader décédé particulièrement vénéré par les mormons de Bountiful, pour expliquer la persécution vécue:

Il est plaisant de noter que nous sommes dignes d'un peu de persécution de temps en temps afin de nous soumettre pour obtenir l'esprit du Seigneur avec nous assez pour détourner l'esprit de destruction qui est dans le cœur de ceux qui cherchent à nous chasser de notre place. Tous les enfants de Dieu ont le droit de vivre sur la terre. Ils ont mérité une place ici, ou Il ne les aurait pas envoyés ici. Nous avons tous le droit de respirer l'air frais qu'il nous a donné. Toutes ces grâces appartiennent aux enfants des hommes, qu'ils respectent les commandements de Dieu ou qu'ils ne les respectent pas. Le Seigneur aime ses enfants, et il est longanime et indulgent. (Leroy Johnson, cité par Blackmore, 2004: 1)

Il est intéressant de noter que, dans ce sermon, Leroy Johnson suggère que la persécution n'est adressée qu'à ceux qui en sont dignes: «il est plaisant de noter que nous sommes dignes d'un peu de persécution». Lorsqu'il poursuit en invitant ses fidèles à la prière afin de chasser l'esprit de destruction du cœur de ceux qui les dénoncent, il se trouve à les rassurer du même coup puisqu'il les désigne comme obéissant aux commandements de Dieu, contrairement à ceux qui dénoncent leurs pratiques. Une invitation au clivage entre les membres de l'Église et ceux qui les critiquent est subtilement proposée dans cet extrait de Blackmore malgré ses affirmations que le Seigneur, indulgent, aime tous ses enfants. En effet, comme le notent certains auteurs (Hogg, 2001; Ellemers et al, 2002; Smurda et al., 2006), une certaine stéréotypie est notée dans la façon avec laquelle il désigne ceux dont il se sentent menacés en parlant de: «l'esprit de destruction qui est dans le cœur de ceux qui cherchent à nous chasser de notre place». Tel que Hogg (2001) le propose, le désir de sauvegarder l'estime personnelle positive des membres de son Église peut également se profiler dans cette adresse de Blakmore à ses fidèles. Il n'est donc guère surprenant qu'une stratégie de retrait silencieux ait d'abord été vue comme la réaction la plus appropriée aux allégations à leur endroit. Au cours de cette période, le groupe évite d'autant plus facilement les interactions avec la communauté élargie, se réfugiant dans une position de retrait, qu'ils se sentent 
au-dessus des allégations formulées à leur endroit. Ainsi, le fait que les allégations soient conceptualisées comme autant de manifestations de persécution qui ne les touchent pas donne une idée de comment ces allégations servent à les confirmer dans la mythologie propre à leur religion. Par conséquent, ces allégations servent à bien marquer leur différence d'avec ceux de la communauté extérieure qui ne suivent pas les commandements de Dieu, dans les mots de Leroy Jonhson, différence dont ils sont fiers. Certes les allégations donnent lieu à des visites d'agents de la GRC et d'intervenants des services de protection des enfants, mais elles ne provoquent pas la naissance de relations conflictuelles avec ces agents de contrôle social, probablement parce que les membres ne s'en sentent pas menacés: "J'ai une bonne relation avec la police, je discute avec eux.» (Julia, avril 2005)

Il se dégage de cet extrait d'entrevue l'image d'une certaine complicité entre l'agent de la GRC et Julia, image qui se dégage également du discours d'autres participants. La patience tolérante semble teinter la tonalité affective utilisée devant les allégations et leurs conséquences, soit la visite accrue d'agents de contrôle social. La stratégie de retrait et l'absence de réfutation ou de réponse directe aux allégations véhiculées par les médias constituent au cours de cette première période une réaction conçue comme l'illustration de leur sérénité devant le caractère mensonger et malicieux qu'ils accordent à ceux et à celles qui soutiennent ces allégations.

\section{La contestation : 2004-2006}

Un conflit avec Warren Jeffs, le leader de la Fundamentalist Church of Jesus Christ Latter-day Saints, dont le siège social est à Colorado City, a provoqué l'excommunication du leader du groupe de Bountiful, Winston Blackmore, en 2002. Un nombre important de mormons a choisi de suivre ce dernier, ce qui provoqua la scission de la communauté de Bountiful en deux groupes de mormons fondamentalistes. Cette scission a eu comme conséquence de réduire considérablement les ressources humaines du groupe de Winston Blackmore, auquel appartiennent les participants à cette recherche. Pour pallier la perte de capital humain, les membres ont choisi de s'inscrire à des activités sportives et récréatives à l'extérieur de la communauté. Ils ont aussi commencé à fréquenter de façon régulière la ville de Creston, située près de Bountiful. 
Alors que les relations avec les citoyens de Creston sont décrites par les participants comme ayant été harmonieuses jusqu'alors, la publication en 2004 d'un livre écrit par une ancienne membre de la communauté de Bountiful (Palmer et Perrin, 2004) modifie le rapport entre ces deux groupes d'acteurs. Des réactions décrites par les participants laissent transparaître une certaine perplexité chez les habitants de Creston:

Je pense que probablement au cours des six derniers mois à un an, quand on entre dans un commerce, les gens ne savent pas comment agir autour de nous. Ils sont comme paranoïaques, ils ne savent pas comment agir avec moi et je ne sais pas pourquoi c'est ainsi. Ils ont entendu ces choses et ils ne savent pas comment agir. Ils ne savent pas s'ils doivent être aimables ou grossiers ou bien s'ils doivent nous éviter. (Charity, avril 2005)

D'autres réactions des habitants de Creston semblent plus hostiles. Les femmes mormones se sentent jugées:

Ils [les habitants de Creston] ont certainement changé la façon dont ils nous servent, la façon dont ils s'adressent à nous, ce n'est pas tout le monde, mais plus de gens. Il devenait évident ce que ces gens pensaient de nous. Et une femme [mormone] m'a dit: «Je sens lorsque je marche dans la rue que je suis une femme stupide, sans éducation, une femme victime de lavage du cerveau.»Et alors nous avons commencé à nous sentir ainsi, et c'était accablant pour nous. (Nicole, avril 2005)

Les mots stupide, sans éducation et victime de lavage du cerveau créent une image qui renvoie à l'idée d'une femme subjuguée, totalement sous la domination des hommes. Cette image rend compte aussi du sentiment d'être disqualifiée socialement qu'exprime Nicole (avril, 2005). Le fait d'être mormon et de le paraître ne transparaît pas dans cet extrait comme étant quelque chose de souhaitable ou dont on peut être fier. L'illusion de pouvoir vivre sans se soucier de l'autre est rompue. Plutôt, le regard de l'autre devient source d'une détresse importante, cela est même accablant (Nicole, avril 2005). L'effet de la stigmatisation se dégage de cet extrait, comme cela a été décrit par certains auteurs (Jenkins 1994; Randall-Noblitt et Penskin, 2000). Il semble ainsi que les allégations pouvaient être traitées avec le silence du mépris tant qu'elles étaient le fait de lointains journalistes et affectaient peu le regard que l'autre proche porte sur eux. Toutefois, la parution de cette autobiographie, en venant soutenir et illustrer nombre d'allégations, aurait provoqué un changement chez ceux-là mêmes avec lesquels ils interagissent le plus. 
Quelques participantes soulignent notamment comment la vie devient difficile pour les enfants et les adolescents, qui se sentiraient stigmatisés en raison de leur habillement et de leur allure, qui les distinguent des autres:

Je suis vraiment désolée pour les jeunes enfants maintenant qu'ils vivent une telle lutte et je sais qu'elle vient de la persécution. Ils ne veulent pas être tellement vus... parce que lorsqu'ils vont en ville ils sont ridiculisés parce qu'ils sont différents. Ils veulent s'intégrer. (Judith, avril 2005)

Ma fille est actuellement adolescente, elle a juste 15 ans, elle va avoir 15 ans. Elle veut faire des choses différentes de celles que je voulais faire. Elle désire des choses différentes de celles que nous faisons, parce qu'elle ne veut pas que les gens la voient comme l'une de ces personnes qui sont tellement dévalorisées dans les médias. Et elle veut faire plus de ces choses que les adolescents du monde font. Par exemple, je n'ai jamais eu de vernis à ongles ou du maquillage lorsque je grandissais, cela ne faisait juste pas une partie de ma vie, nous ne prenions pas le temps de le faire. Maintenant, ils veulent être comme des adolescents normaux et parfois même rejeter nos croyances fondamentalistes, c'est parfois une menace à notre façon de vivre! (Madeleine, avril 2005)

Non seulement les problèmes des enfants sont-ils ainsi attribués aux effets de la persécution dont ils seraient victimes de la part des habitants de Creston, mais les adolescents sont vus comme à risque de rejeter les croyances mormones sous l'effet du regard des autres sur eux. Alors qu'auparavant les allégations ne semblaient pas les toucher, l'effet de la publication du livre de Debbie Palmer sur les gens qui les côtoient est même vu comme une menace à la transmission de leur foi. Désormais les mormons de Bountiful se sentent directement interpellés:

Peu importe ce qu'est Deb [Debbie Palmer] ou peu importe ce que vous croyez qu'elle est, le fait est qu'elle a dit des choses vraiment blessantes à propos des gens de Bountiful. (Jérôme, janvier 2006)

Alors qu'auparavant les allégations confirmaient aux mormons qu'ils étaient certes différents, mais meilleurs que les «autres», la publication du livre de Debbie Palmer a transformé ce qui pourrait être désigné comme un sentiment de persécution acceptable en un sentiment de victimisation, voire d'aliénation sociale. Les allégations conduisent désormais les membres à se sentir stigmatisés beaucoup plus clairement en raison de leur différence. Les changements dans le regard des autres, comme la peur possible de voir les instances publiques accroître leurs 
interventions, amènent une modification de leur réaction aux allégations :

Parce que c'est fou et si vous ne répondez d'aucune façon, alors ils doivent inventer des histoires et dire que vous avez refusé de leur parler. Donc nous sommes allées $[\ldots]$. Nous avions l'habitude d'essayer de ne parler à personne et la grande théorie derrière, cela était: si nous ne disons rien, ils n'auront rien de négatif à publier, à dire à notre propos, mais ce n'était pas vrai. Ils peuvent parler aux gens qui ne nous aiment pas et toujours parler et alors ils inventent une histoire avec cette information et ils n'ont rien avec laquelle la comparer ou l'argumenter. (Judith, avril 2005)

Plusieurs actions sont entreprises, notamment, certains tentent de parler aux médias malgré la frustration ressentie de ne pas être écoutés:

Nous avons également tenté de parler aux gens et aux journalistes, nous étions réellement disposés à discuter et à les aider à comprendre, mais ils sont venus avec leurs objectifs, ils ne veulent pas la vérité, ils veulent du sensationnalisme. Le sensationnalisme, c'est ce qui vend. C'est uniquement ce qu'ils veulent. Si nous disons quelque chose, nous ne le voyons pas dans le journal et je suis indignée par cela. (Kathleen, avril 2005)

Les médias viennent et ils sont amicaux, ils sont ouverts, ils sont charmants et ils se retournent et ils ne sont pas intéressés à ce que nous disons, seulement à le comparer avec ce que quelqu'un d'autre dit. Et ils donnent toujours à quelqu'un d'autre le dernier mot. Comment vous le dites, comment le présenter. Ils ne sont pas nos amis. Donc, comment procédons-nous pour transmettre cette information à l'extérieur, la vérité? (Mark, avril 2005)

Ce dernier extrait illustre comment les membres de la communauté de Bountiful se sentent impuissants à transmettre leurs messages aux médias. Simplement réfuter les allégations n'apparaît donc plus comme une solution efficace. D'autres choisissent de participer à des forums de discussion Web, ce qui leur offre plus de contrôle sur le message transmis. La contribution suivante montre bien, notamment par l'utilisation des majuscules, la colère et la blessure ressenties par Joy. Il se dégage également de cet extrait le sentiment d'être protégée dans la tourmente actuelle par la mission religieuse qu'elle partage avec ses coreligionnaires en faisant le Vrai Travail de Dieu [sic]:

Je suis allée sur le lien et j'ai lu Tous [sic] les articles de recherche par l'ensemble des Professeurs [sic], Universités [sic], toutes financées [sic] avec 150000 dollars de l'argent des payeurs de taxes. Joseph [interlocuteur], 
imagine que Deb [Debbie Palmer] a suffisamment de pouvoir, d'influence, et de CERVELLE [sic] pour faire en sorte que ça se produise. Je suppose alors que nous avons un problème. Même en considérant le fait que TOUTES LES RECOMMANDATIONS DE CES RECHERCHES [sic] étaient fortes dans leur reconnaissance que les gens de Bountiful, et tous ceux qui considèrent la polygamie comme faisant partie «d'une religion, culture ou d'un style de vie», doivent être traités avec dignité et respect. Ils reconnaissent également qu'il y a vraiment peu d'accès à de l'information JUSTE [sic] concernant ce qui se produit réellement dans des communautés comme Bountiful. Donc la porte, le portail, et l'accès à chacun des ministères gouvernementaux sont GRAND OUVERTS [sic] pour la meilleure information HONNÊTE [sic] que nous pouvons donner à propos du Vrai Travail de Dieu [sic]. (Joy, janvier 2006)

Un groupe de femmes choisissent quant à elles de participer à divers congrès dans les villes de Winnipeg et de Vancouver où Debbie Palmer est conférencière. Cette nouvelle manière de répondre aux allégations s'apparente à la réaction de contestation décrite par Bromley (2002) et Wright (2002). Cette contestation prend notamment la forme d'une conférence organisée en 2005 dans la ville de Creston, le Summmit on Polygamy, ouvert au grand public et dont la tenue est abondamment publicisée:

Ce que j'ai décidé, c'est que nous ne pouvons plus rester immobiles, les gens qui formulent les allégations, nous devons les suivre et dire: «Ne les laissez pas parler pour moi!» Je vais à Vancouver lundi pour faire cela. Je vais être là parce qu'ils vont parler de nous et j’ai décidé que... j'ai mes enfants... j'ai une vie à vivre, mais mes enfants et ma vie n'ont plus de valeur si je reste silencieuse parce que le silence influence tellement nos vies. J'ai décidé d'aller à Vancouver pour me tenir debout et pour dire: «Je parle pour moi-même.» (Nicole, avril 2005)

Avec le sommet, je savais que cela n'allait pas faire une différence, mais je crois que le but premier était... Il y avait les gens qui ont été bons pour nous, qui nous ont accepté, donc on se devait de les aider à nous comprendre un peu mieux et pour les autres, leur laisser savoir que nous sommes humains. (Judith, avril 2005)

Organisée par des femmes du groupe et regroupant près de cent cinquante membres dans l'assistance, la conférence vise à éduquer les non-membres et à contrer les allégations dont ils se sentent victimes. Malgré le pessimisme manifesté par Judith, le désir de se faire connaitre se dégage de ses propos. Le désir de montrer qu'ils sont humains: «leur 
laisser savoir que nous sommes humains» (Judith, avril 2005) indique aussi jusqu'à quel point elle se sent rejetée et stigmatisée par les allégations dont elle se sent victime. Laccent est donc mis autant sur les différences que sur ce qui est commun, par exemple le fait d'être tous humains, mais aussi le message transmis semble viser à faire des mises au point:

Et tu sais, nous disions: «Ceci [mariage à l'âge de 15 ans], cela n'arrivera jamais plus!» Les filles ne se marieront jamais plus avant l'âge de 18 ans; dans une situation de pluralité, non! Si une fille désire se marier avec un homme plus jeune, il n'y a rien de mal là-dedans. Je pense que c'est une sage chose pour les filles de le faire. (Charity, avril 2005)

Ainsi une des allégations fréquemment véhiculées, le mariage précoce des adolescentes à des hommes polygames beaucoup plus âgés qu'elles, est prise de front et une promesse de rectifier cette situation est faite. Dans ce sens, il peut être dit que les allégations ont porté leurs fruits, permettant de protéger de jeunes adolescentes du mariage polygame. Lallégation par médias et témoignages autobiographiques interposés semble avoir permis un échange, une communication réelle, bénéfique pour les adolescentes de la communauté. Le besoin du groupe d'être accepté par la société environnante semble beaucoup plus important que ce que le retrait silencieux permettait de supposer. Il semble bien que ce soit une chose d'ignorer un autre vu comme lointain et abstrait en espérant qu'il finisse par nous oublier et une autre de se sentir directement l'objet d'opprobre. En ce sens, la contestation manifestée par les mormons de Bountiful ne se compare d'aucune façon aux mouvements de confrontation décrits par nombre d'auteurs ((Larkin, 1991; Casoni, 2000; Bromley, 2002; Wright, 2002). Judith exprime ce besoin de communication avec des gens extérieurs à son groupe assez clairement dans l'extrait suivant:

Je veux juste rencontrer quelqu'un, me faire des amis pour avoir quelqu'un quelque part qui nous connaît au moins un peu et qui peut nous aider à faire cesser ou à faire dévier un peu de tension projetée là-bas sur nous. (Judith, avril 2005)

Le besoin exprimé par Judith de prendre contact avec quelqu'un extérieur à sa communauté qui puisse la croire et la soutenir rend bien le sentiment d'isolement et de stigmatisation dont elle se sent victime. Le temps du retrait silencieux semble bien révolu. Devant le jugement moral négatif qu'ils ressentent de la part de l'autre proche, les mormons 
abandonnent le retrait silencieux pour une attitude beaucoup plus axée sur la communication et l'échange, fut-elle surtout faite de réfutations et de contestation. Le fait de se sentir trahis par l'autobiographie de Debbie Palmer, mais surtout le fait de devenir des êtres discrédités dans le regard des habitants de Creston causent une détresse qui les pousse à l'action.

À cet effet, la mobilisation des femmes du groupe dans la contestation des allégations est intéressante à noter, puisque ce sont elles qui sont vues comme les premières victimes de la communauté. La réaction de contestation est, de fait, très présente puisqu'à partir de 2004, les mormons du groupe de Winston Blackmore sont de toutes les tribunes et profitent de ces occasions pour réfuter les allégations qui pèsent contre eux. Cependant, la contestation s'accompagne aussi de modifications au sein de la communauté qui visent à l'accommodement aux valeurs de la société. La plus importante modification consiste assurément en la règle au sein de la communauté selon laquelle désormais aucune adolescente de moins de 18 ans ne pourra se marier avec un homme polygame plus âgé qu'elle. Le second changement concerne l'utilisation de punitions corporelles contre les enfants. D'ailleurs, dans le but d'éviter le glissement de la punition corporelle aux sévices physiques, les femmes rencontrées disent en avoir abandonné l'usage. La réaction aux allégations a ainsi amené le groupe à se rapprocher des valeurs de la société environnante après une première réaction de retrait et d'isolement. Par conséquent, une certaine pression médiatique semble avoir eu un effet positif, contrairement aux thèses de Bromley (2002), Hall (1995) et Wright (2002) chez les mormons fondamentalistes à l'étude.

\section{Conclusion}

Le questionnement sur la réaction des groupes religieux minoritaires devant la pression médiatique et celle des intervenants sociaux lorsque des allégations d'entorses aux lois sont formulées n'en est qu'à ses balbutiements. De fait, le constat que deux types différents de stratégie ont été observés chez les mormons fondamentalistes de Bountiful, à la suite des allégations dont ils sont l'objet, montre l'intérêt d'étudier temporellement les réactions des groupes minoritaires lorsqu'ils subissent une pression de la part de la société environnante. Dans le cas du 
groupe à l'étude, il s'avère que les membres sont passés d'une attitude de retrait silencieux, qui les marginalisait assurément, à une de contestation qui a le mérite de les engager dans le discours avec des acteurs en dehors de leur milieu, rompant ainsi quelque peu l'isolement protecteur auparavant érigé en barrière contre les contacts.

Toutefois, il y a lieu de se demander comment le groupe réagirait si des accusations criminelles étaient portées contre certains membres à la suite de l'enquête instituée par le Procureur général de la ColombieBritannique. Cette question trouvera peut-être une réponse plus rapidement que prévue, dans l'éventualité où la perquisition récente de la clinique médicale de la communauté par la GRC permettrait d'établir que des jeunes filles auraient donné naissance alors qu'elles étaient mariées à des hommes plus âgés qu'elles, puisque de telles preuves pourraient résulter en des accusations de détournement de mineures ou d'agressions sexuelles contre ces hommes. De telles accusations résulteraient-elles en un retour à une attitude refermée qui isole les mormons du regard de l'autre? Le dialogue en serait-il pour autant terminé? En effet, il est plus facile de protéger des êtres vulnérables dans un contexte où la communauté n'est pas totalement refermée sur elle-même. Tant qu'un dialogue est possible, l'atteinte des objectifs sociaux comme la protection des enfants, ou celle des femmes en situation de violence conjugale, par exemple, est beaucoup plus facile. Remplir leurs obligations légales est certes plus facile pour les intervenants sociaux dans un contexte de dialogue, surtout lorsque l'idéologie du groupe préconise l'acceptation des épreuves, dont celles liées à des mauvais traitements, comme une preuve d'amour pour Dieu. Il reste à voir aussi comment le gouvernement de la Colombie-Britannique gérera ce dossier. Il est à souhaiter qu'il fasse preuve de savoir-faire sans pour autant renoncer à ses responsabilités, notamment dans la protection des enfants.

\section{Références}

Armstrong, J. (30 septembre 2004). Mother fears sect's hold over daughter: Fundamentalist group from Bountiful, B.-C., is building an isolated compound in Texas. The Globe and Mail, p. A-19.

- (27 octobre 2005). Bountiful's children break out. Students from fundamentalist community bring new life to declining secular school. The Globe and Mail, p. A-4. <http://www.theglobeandmail.com/servlet/ArticleNews/TPStory/LAC/20051027/BCBOUNTIFUL27/TPEducation/> 
Barker, E. (2002). Watching for violence: a comparative analysis of the roles of five types of cult-watching groups. In D. G. Bromley \& G. Melton (eds.), Cults, religion of violence (123-148). Cambridge: Cambridge University Press.

Beaman, L. (2004). Church, State and the Legal Interpretation of Polygamy in Canada. Nova Religio, 8 (1), 20-38.

Bell, S. (12 juillet 2002). Word is out' Canada is a safe haven Prosecutors fear any case would fail Charter of Rights test. National Post, p. A-2.

Beiser, V. (26 juillet 1999). The Perils of Polygamy. Maclean's, p. 32.

Betty, J. (4 septembre 2004). Rights tribunal agrees to hear polygamy case: Four ministries accused of failing to protect the girls and women of Bountiful. Vancouver Sun, <http://www.rickross.com/reference/polygamy/polygamy247.html>

Bistline, G. B. (2004). The Polygamists : A History of Colorado City Arizona. Utah: Agreka.

Blackmore, W. (2004). A Right to Live. North Star Chronicles, 2 (9), p.1.

Bramham, D. (29 mai 2004). B. C. is a safe haven for polygamists: But what about the women and children? Vancouver Sun, p. 7.

- (4 juin 2004) Utah A-G Declares War on Polygamy: B. V. Ignores Cult is Focus of Attention in U. S. Vancouver Sun, p. 4.

- (15 juin 2004). Cabinet talks a signal of hope for child brides of Bountiful: A letter detailing abuse behind the cult's veil has spurred the attorneygeneral to action. Vancouver Sun, <http://www.childbrides.org/canada_ cabinet_talks_signal_of_hope.html>

- (21 juillet 2004). Civil libertarians want Bountiful inquiry: For years, government has ignored reports of sex abuse, exploitation, denial of rights. Vancouver Sun, p. 11.

- (7 août 2004). The Many Wives of Bountiful B. C.'S Attorney-General probing sect's polygamy, Alledge Traffiking of Teenage Girls to U. S. Vancouver Sun, p. 7.

- (15 décembre 2004). Bountiful schools get public funds, but government scrutiny is suspect. Vancouver Sun, p. 16.

- (13 octobre 2005). Woman who left U. S. polygamous cult speaks at Vancouver film fest. Brandon Sun, <http://www.religionnewsblog.com/12454/ woman-who-left-us-polygamous-cult-speaks-at-vancouver-film-fest $>$

Brewin, A. (2004). Women's Equality and Religious Freedom Consultation Report. West Coast: LEAF.

British Colombia Teachers' Federation (5 juillet 2004). Board Meeting: Report. Lister: BCTF.

Bromley, D. G. (2001) A tale of two theories: brainwashing and conversion as competing political narratives. In B, Zablocki \& T. Robbins (eds.), Misunderstanding cults: Searching for objectivity in a controversial field (318-348). Toronto: University of Toronto Press.

- (2002). Dramatic Denouements. In D. G. Bromley \& G. Melton (eds.), Cults, religion $\sigma$ violence (11-41). Cambridge: Cambridge University Press. 
Cart, J. (18 août 2002). As activists gather, they say state's inaction on the issue allows sexual assaults, child abuse, Welfare fraud to go unchecked. Los Angeles Times, <http://www.rickross.com/reference/polygamy/polygamy84.html>

Casoni, D. (2000). Du paradis à l'enfer : étude des mécanismes psychologiques associés aux dérives sectaires. In J. Duhaime (ed.). La peur des sectes. Montréal: Éditions Fides.

Campbell, A., Bala, N., Duvall-Antonacopoulos, K., MacRae, L., Paetsch, M., Bailey, J., Baines, B., Amani, B., \& Kaufman, A. (2005). La polygamie au Canada: conséquences juridiques et sociales pour les femmes et les enfants. Recueil de rapports de recherche en matière de politiques. Edmonton: The Alberta Civil Liberties Research Center.

Chattaway. P. T. (7 juillet 2004). B. C. urged to charge polygamists, Canadian Christianity,

<http://www.canadianchristianity.com/cgibin/na.cgi?nationalupdates/ 040707polygamists>

Committee on Polygamous Issues (1993). Life in Bountiful - A report on the lifestyle of a polygamous community, (préparé pour le Committee on Polygamous Issues, financé par le Ministry of Women's Equality (C.-B.). Vancouver : CPI.

Cook, R. (2006). La polygynie et les obligations du Canada en vertu du droit international en matière de droits de la personne. Toronto: Ministère de la Justice du Canada.

D’Amour, M. (27 juillet 2004). Sect greets abuse probe: Polygamist leader says group will co-operate. Calgary Sun, p. 3.

- ( $1^{\text {er }}$ août 2004). Polygamists defend lifestyle: Commune and town of Creston co-exist in a delicate economic balance. Calgary Sun,

<http://www.religionnewsblog.com/8106/polygamists-defend-lifestyle>

- (2 août 2004). Human smuggling denied Critic charges girls trafficked as breeding stock. Calgary Sun, <http://www.rickross.com/reference/ polygamy/polygamy231.html>

Dawson, F (22 septembre 2000). Polygamists scoop teens Girls allegedly being wed to elders of church with communes in U. S. The Province, p. 3.

Dougherty, J. (13 mars 2003). Bound by Fear: Polygamy in Arizona For decades the state has let a feudal colony of fundamentalist Mormons force underage girls into illegal polygamous marriages. Phoenix NewTimes.com $<$ http://www.phoenixnewtimes.com/2003-03-13/news/bound-by-fearpolygamy-in-arizona/>

- (10 avril 2003). Polygamy in Arizona: The Wages of Sin. Phoenix New Times, p. A-5.

- (21 août 2003). Utah Targets Polyg Prophet While Arizona autorities languish, the Utah Attorney General's Office focuses on the leader of the fundamentalist Mormon Church. Phoenix New Times, p. A-7.

Ellemers, N., Spears, R., \& Doosje, B. (2002). Self and Social Identity. Annual Review of Psychology, 53, 161-186. 
Elsworth, C. (5 août 2004). North America - Canada - FLDS «Investigation launched into polygamous sect dubbed 'Canada's dirty little secret» Telegraph, p. A-13.

Euringer, A. (18 novembre 2004). Leaving Bountiful: A rare, intimate interview with the rebellious first wife of the polygamous B. C. Mormon community's longtime leader. The Tyee, <http://thetyee.ca/News/2004/11/18/Leaving Bountiful/>

- (26 mai 2006). Talking to 'Lost Boys' of Bountiful A conversation with two trying to make it outside. The Tyee, <http://thetyee.ca/News/2006/ 05/26/Bountiful/>

Frazier, D., \& Florio, G. (16 juillet 2005). Principle' is Bedrock Law; Men Take Multiple Wives; Women are Taught to Comply. Rocky Mountain News (Denver), <http://www.rickross.com/reference/polygamy/polygamy362. html>

French, J. (12 juin 2004). Cults abusive to women, children. Polygamist colonies. Edmonton Journal, <http://www.rickross.com/reference/polygamy/ polygamy213.html>

Hall, J. R. (1995). People's Temple. In T. Miller (ed.), America's alternative religions (303-311). Albany: State University of New York Press.

Hall, J. R., \& Schuyler, P. D. (1997). The Mystical apocalypse of the Solar Temple. In T. Robbins \& S.J. Palmer (eds.), Millennium, messiah, and mayhem: contemporary apocalyptic movement (247-260). New York: Routledge.

Hall, J. R, Schuyler, P. D., \& Trinh, S. (2000). Apocalypse Observed. Religous movements and violence in North America, Europe and Japon. New York: Routledge, Taylor \& Francis Group.

Hogg, M. A. (2001). A Social Identity Theory of Leadership. Personality and Social Psychology Review, 5 (3), 184-200.

Jacobs, M. (12 juin 2004). Blind eye turned to forced polygamy. The Edmonton Sun, p. A-5.

- (21 mai 2006). Put polygamy to constitutional test. The Edmonton Sun, <http://pages.usherbrooke.ca/sodrus/pdf/pdf_dossiers/Polygamie2. pdf>

Jenkins, P. (1994). Using Murder: The Social Construction of Serial Homicide. New York: Preago.

Kent, S. (2006). A Matter of Principle Fundamentalist Mormon Polygamy, Children, and Human Rights Debates. Nova Religio, 10 (1), 7-29.

La Presse (27 décembre 1990). Dénonciation d'une secte pratiquant la polygamie. La Presse, p. A-19.

Latkin, C. (1991). From device to vice: social control and intergroup conflict at Rajneeshpuram. Sociological Analysis, 52 (4), 363-378.

Lifton, R. J. (1999). Destroying the World to Save It. Aum Shinrikyo Apocalyptic Violence and the New Global Terrorism. New York: Owl Books.

Luhtanen, M., McKay-Panos, L., \& Seaman, B. (2005). Distincts et inégaux: les femmes et les enfants de la polygamie. Edmonton: The Alberta Civil Liberties Research Center. 
Mac Queen, K. (13 décembre 2004). The battle for Bountiful, Religion; Polygamy, radicalism and a fight for hearts and minds: a Mormon sect's power struggle. Macleans Magazine, <http://www.rickross.com/reference/polygamy/polygamy288.html>

Matas, R. (24 septembre 1990). Community Practices Polygamy. The Globe and Mail, p. A-1 et A-8.

- (19 novembre 2002). Woman to bring suit against Mormon Church. The Globe and Mail, <http://www.rickross.com/reference/polygamy/polygamy96.html>

- (4 avril 2003). Bishop loses Bountiful school case. The Globe and Mail, <http://www.rickross.com/reference/polygamy/polygamy109.html>

- (2005). Preventing sexual abuse in a polygamous community. Beyond Borders, p.1-18.

Mayer, J. F. (2001). Cults, Violence and Religious Terrorism: An International Perspective. Studies in Conflict $\mathfrak{*}$ Terrorism, 24 (5), 361-376.

Nichols, J. (19 octobre 2003). Women in polygamist marriages fight back. Arizona Republic, <http://www.rickross.com/reference/polygamy/polygamy142.html>

Oziewicz, E. (9 décembre 2000). Bountiful's troubling tradition Men in this quiet B. C. community aren't limited to just one mate. The Globe and Mail, $<$ http://www.rickross.com/reference/polygamy/polygamy48.html>

Palmer, D., \& Perrin, D. (2004). Keep Sweet: Children of Polygamy. Lister: Dave's Press.

Pelland, M-A, Casoni, D. (2009, à paraître). Portrait des mormons fondamentalistes de l'ouest canadien. De Joseph Smith à Winston Blackmore. In M. Geoffroy \& J. G. Vaillancourt. La religion extrême. Montréal: Médiaspaul.

Pelland, M-A, Casoni, D. (soumis). Eux et nous. Vicissitudes de l'identité sociale chez un groupe de mormons fondamentalistes. Déviance et Société.

Quinn, M. D. (1994). The Mormon Hierarchy: Origins of Power. Salt Lake City: Signature Book.

Randall Noblitt, \& J. Perskin. (2000). Cult and Ritual Abuse: Its History, Anthropology, and Recent Discovery in Contemporary America. New York: Praeger.

Remington, R. (27 septembre 2000). Sect leader not worried about polygamy probe Reports of teen brides. National Post, p. A-11.

Robbins, T. (2002). Sources of Volatility in Religious Movements. Dans D.G. Bromley \& G. Melton (eds.), Cults, religion o violence (57-79). Cambridge: Cambridge University Press.

Russell, J. (2004). Annual Report. British Columbia Civil Liberties Association, <www.bccla.org/newsletter/BCCLA \%20AR\%202004\%20web.pdf>

Shurtleff, M., \& Goddard, T. (2006). The Primer. Helping Victims of Domestic Violence and Child Abuse in Polygamous Communities. Utha: Government of Utah, <http://www.attorneygeneral.utah.gov/polygamy/The_Primer.pdf. p.1-56> 
Smurda, J. D., Wittig, M. A., \& Gokalp, G. (2006). Effects of Threat to a Valued Social Identity on Implicit Self-Esteem and Discrimination. Group Processes $\sigma$ Intergroup Relations, 9 (2) 181-197.

Stick, F. (5 mars 2002). Canadian Polygamist Let Off the Hook-Again Governement says the Polygamy Law violates religious Freedoms. Christian Week, p. A-14<www.Christianweek.org>

Van Wagoner, R. S. (1989). Mormon Polygamy: A History. Second Edition. Salt Lake City: Signature Books.

Weatherbee, S. (16 août 1993). The practice of polygamy: a Mormon colony stirs a B.C. controversy. Maclean's, < http://thehopeorg.org/canada_Mac_ plig_BC_controversy.html>

Weland, M. (24 novembre 2004). Rift in FLDS Church raises local fears. Kootenai Valley Press, <http://www.rickross.com/reference/polygamy/polygamy279.html>

Wilson, B. R. (1991). The social Dimensions of Sectarianism. Oxford: Clarendon Press.

Woods, D. (4 août 2001). Bountiful, B.C. Saturday Night, <http://www.rickross.com/reference/polygamy/polygamy65.html>

Wright, S. A. (2002). Public agency involvement in government - religious movement confrontations. In D. G. Bromley \& G. Melton (eds.), Cults, religion $\mathfrak{F}$ violence (102-122). Cambridge: Cambridge University Press.

ABSTRACT - This article seeks to understand the impact of allegations of illegal conduct on a group of polygamous Fundamentalist Mormons established in Bountiful, British Columbia. These allegations include the illegal passage of young women between Canada and the United States in order to provide older polygamous men with young wives, as well as the sexual and physical abuse of children, adolescents and women. Interviews with group members, analysis of the writings of the group and of the discussions held by members of the group on a WEB Forum, as well as a review of the principal Canadian and American periodicals and newspapers from 1990 to 2006 are used to identify the allegations and outline the group's reactions. Two distinct types of reactions to the allegations are identified, the first being a silent retreat, which was followed by a reaction of contestation. These reactions are described, analysed and illustrated with the help of extracts from interviews and Web exchanges.

RESUMEN - Este artículo tiene por objetivo comprender el impacto de diversos alegatos relativos a violaciones a la ley sobre un grupo de mormones fundamentalistas polígamos establecidos en Bountiful, Columbia Británica. Dichos alegatos van del tráfico de mujeres jóvenes entre Canadá y Estados Unidos con el fin de casaras con hombres polígamos mayores que ellas a abusos físicos y sexuales sobre menores, adolescentes y mujeres. Entrevistas a miembros de este grupo asi como un análisis documental del periódico de la comunidad, de intercambios en el foro de discusión web del grupo y de los principales diarios y periódicos canadienses y estadounidenses de 1990 a 2006 han permitido documentar dichos alegatos e identificar las reacciones del grupo ante ellos. Dos tipos de reacción fueron identificados: en un primer tiempo, un repliegue silencioso del grupo; en un segundo tiempo, una actitud de protesta predominó. Estas reacciones son descritas, analizadas e ilustradas gracias a extractos de entrevistas e intercambios web. 